\title{
Full snapshot reconstruction in hybrid architecture antenna arrays
}

\author{
Maria Trigka* (1), Christos Mavrokefalidis and Kostas Berberidis
}

*Correspondence:

trigka@ceid.upatras.gr

Department of Computer

Engineering and Informatics,

University of Patras, Campus

Rio, 26504 Patra, Greece

\begin{abstract}
In the context of this research work, we study the so-called problem of full snapshot reconstruction in hybrid antenna array structures that are utilized in mmWave communication systems. It enables the recovery of the snapshots that would have been obtained if a conventional (non-hybrid) uniform linear antenna array was employed. The problem is considered at the receiver side where the hybrid architecture exploits in a novel way the antenna elements of a uniform linear array. To this end, the recommended scheme is properly designed so as to be applicable to overlapping and nonoverlapping architectures. Moreover, the full snapshot recoverability is addressed for two cases, namely for time-varying and constant signal sources. Simulation results are also presented to illustrate the consistency between the theoretically predicted behaviors and the simulated results, and the performance of the proposed scheme in terms angle-of-arrival estimation, when compared to the conventional MUSIC algorithm and a recently proposed hybrid version of MUSIC (H-MUSIC).
\end{abstract}

Keywords: 5G, MmWave, Hybrid architectures, AoA estimation

\section{Introduction}

Communications in the millimeter wave (mmWave) spectrum are bringing a new era for the next generations of wireless and cellular telecommunication systems [1-3]. It will enable gigabit-per-second data rates thanks to the large bandwidth of available frequencies (namely, from $30 \mathrm{GHz}$ to $300 \mathrm{GHz}$, and also $\mathrm{THz}$ ), addressing the emerging demand of cellular networks for higher data rate. Due to the short wavelength of mmWave, more antenna elements can be packed into the same physical area, enabling the use of massive multiple-input and multiple-output (MIMO) antenna arrays at both the transmitter and the receiver. The leverage of mmWave with the promising massive MIMO physical layer technology will increase spectral and power efficiency, transmission throughput and network coverage of the fifth generation (5G) networks ensuring the successful support of a wide variety of emerging applications, such as augmented reality (AR), virtual reality (VR), cloud-based services, smart city, vehicular-to-vehicular (V2V) or vehicular-to-everything (V2X) communication systems for autonomous vehicles, Machine-to-Machine (M2M)/Internet-of-Things (IoT), multimedia, etc. [4].

However, the implementation of a conventional MIMO with a large-scale antenna array and a full digital precoding (combining) approach is still prohibitive. This is author(s) and the source, provide a link to the Creative Commons licence, and indicate if changes were made. The images or other third party material in this article are included in the article's Creative Commons licence, unless indicated otherwise in a credit line to the material. If material is not included in the article's Creative Commons licence and your intended use is not permitted by statutory regulation or exceeds the permitted use, you will need to obtain permission directly from the copyright holder. To view a copy of this licence, visit http:// creativecommons.org/licenses/by/4.0/. 
because each antenna element in the transmitter (receiver) is connected to a digital (analog) to analog (digital) converter and an radio frequency (RF) chain of analog elements. Such an option brings high implementation cost and large power consumption at mmWave band when the antennas are too many. An alternative approach to reduce the number of RF chains is hybrid analog/digital architectures, where the processing is split between an analog and a digital part. The analog part of a hybrid precoder (combiner) can be implemented by employing elements such as phase shifters, switches or lens antenna $[5,6]$, to name some of the most common proposed in the literature. Depending on the involved elements different hardware constraints arise [3]. In the literature, two main MIMO architectures have been proposed [7, 8] for the RF precoding (combining) matrix. In both cases, the aim is to find the optimal number of RF chains thus optimizing the cost, energy consumption and complexity. The first one (see Fig. 3) is called fully connected architecture, where each of the available RF chains is connected to all antenna elements, while, in the second one, the so-called partially connected (see Fig. 2), the involved RF chains are associated with unique non-overlapping subgroups of antenna elements [9]. Assuming a network of phase-shifters, the main characteristics and differences between these architectures are the following [10]: (1) A fully connected network provides full precoding (combining) gain, achieves highly directive transmissions by adjusting the phases of the transmitted signals in all antenna elements with constant modulus phase-shifters, but it has high complexity. (2) In a partially connected network, for each RF chain, only the transmitted signals on the corresponding subset of antennas can be adjusted. For practical reasons, the partially connected structure is often preferable, although it achieves reduced array gain and directivity, proportional to the number of subarrays. Also, it imposes the analog precoder (combiner) to be a block diagonal matrix of unit magnitude nonzero elements. Additionally, it has lower hardware complexity at the cost of beamforming gain, as compared to the fully connected structure. The authors in [11] claim that there is no distinct solution for the hybrid structure that can offer the best trade-off between complexity and performance. Hence, a dynamic structure is needed depending on the application and channel conditions.

Since the wavelength at mmWave is shorter than the one in microwaves, material penetration will incur greater attenuation, thus increasing the significance of line-of-sight (LOS) propagation and reflection. Due to severe path loss, a few reflecting paths could arrive at the receiver [2]. In this case, the channel is strongly sparse in the angle domain. The high propagation attenuation, the increased sensitivity to blockage and the possible mobility of users require mature signal processing techniques and novel insights in architectures and protocols to combat these challenges. Hence, severe propagation losses occurring at the mmWave band could be balanced by hybrid precoding (combining) techniques using massive MIMO technology, in order to provide high antenna array gain, through narrow directional beamforming, and sufficient spatial coverage [10].

The mmWave propagation environment is typically modeled via a geometric channel model which involves the angles of departure $(\mathrm{AoD})$ and the angles of arrival (AoA) at the transmitter and receiver sides, respectively. It is noted, here, that the problem of estimating of AoD/AoA in the frame of array signal processing is closely related to the problem of channel estimation and beamforming in the frame of mmWave wireless communications. The core idea of parametric massive MIMO channel modeling is to 
estimate the involved angles and the respective complex factors, instead of estimating the channel impulse response (communication viewpoint). The AoA estimation has been of particular interest to researchers for several decades and still remains an active area in wireless and mobile communication (e.g., radar, smart antenna) [12].

The AoA estimation algorithms [13, 14] and their variants, such as [15], have been designed to estimate the unknown angles from the received vector for a classical ULA. A beam scanning is applied by a full analog array to search the AoA, while spectrumbased techniques can be employed by a full digital array. Many high-resolution subspace-based methods have been developed, with the most widely used among them due to their simplicity, being the MUSIC (MUltiple Signal Classification) [16-18] and ESPRIT (Estimation of Signal Parameters via Rotational Invariant Techniques) [19, 20] algorithms. Such algorithms exploit the structure of the signal/noise subspaces and estimate the desired angles as the points at which the received signal spectrum is maximized. However, the main drawback of these algorithms is that they are not compatible with the hybrid architectures that are proposed for mmWave transmitters (receivers). Moreover, these algorithms fail to resolve coherent/highly correlated signals which are very common in communication systems due to the multi-path phenomenon, thus their performance is degraded. Many methods have been suggested to treat the coherent case, which are applicable to ULAs as well. The most representative are the Spatial Smoothing (SS) [21] and forward/backward-SS (FB-SS) [22]. The role of spatial smoothing techniques is to decorrelate coherent signals and reconstruct a full-rank source signal covariance matrix.

A solution in [23] focuses on the design of high-resolution AoA algorithms (e.g., of the MUSIC and ESPRIT type) for hybrid architectures assuming coherent source signals, a feature incorporated to the system model and proposed algorithms. Actually, the source signals consist of multi-path copies of a transmitted signal. Nevertheless, the algorithms proposed in [23] cannot handle effectively the coherent case unless the involved channels change at a rate that is close to the snapshot rate, which happens rarely in practice. Several experiments have been conducted to verify the behavior of H-MUSIC in terms of AoA estimation in a coherent environment for different rates of change of the involved channel. Additionally, some preliminary results are presented in our previous works [24, 25], where the AoA estimation problem is also investigated based on the reconstructed ULA snapshot. In both works, the MUSIC algorithm is applied in the reconstructed snapshots to estimate the unknown AoAs. It is pointed out, here, that, differently to [23] and more recent works [6,26-28], this paper summarizes and extends previous findings, providing an alternative approach to the problem. Specifically, unlike previous works which focus on new AoA estimation techniques, in this work, the key idea is to develop techniques which, based on the sampled output of a hybrid array, will reconstruct the snapshots that would have been captured using a conventional (nonhybrid) ULA. Obviously, having reconstructed the full ULA snapshots, any existing AoA estimation algorithm can subsequently be applied, hence, the extensive relevant literature can be still exploited.

As a final note, although the work in this paper has focused on the recoverability of the full snapshot in hybrid antenna arrays using ULAs [29], there are other architectures that could be studied as future extensions of this work. Among different array geometries, 
fractal-wavelet modeling theory [30-39], widely used in antenna theory and its application to small antenna arrays and to the case of mmWave bands would be interesting. Additionally, other antenna array configurations could be studied like co-prime configurations [40]. Finally, lens-antennas, as already have been applied in mmWave communication systems, would be an interesting direction [41].

The main contributions of this paper are briefly the following:

1 An efficient preprocessing scheme is developed that leads to the acquisition of the baseband snapshot irrespective of the hybrid antenna array architecture, as if a nonhybrid antenna array was employed.

2 The new scheme is studied for two typical hybrid receiver architectures implemented through a network of phase shifters that are employed in order to create suitable combinations of the received signals from which the baseband snapshot is retrieved. By maximizing the signal-to-total-noise ratio (STNR) of the restored snapshot, i.e., a signal-dependent noisy term, the proposed scheme determines the optimal values of the phase shifters.

3 The full snapshot recoverability problem is treated for both cases of time-varying and constant source signals during sub-snapshots collection time. A different combiner matrix is applied for each sub-snapshot acquisition.

4. The theoretical findings regarding the proposed optimal reconstruction schemes are verified by extensive simulation experiments.

5 As already mentioned, once the full snapshots are reconstructed via the proposed schemes, then any suitable AoA estimation algorithm can be applied. The coherent case, commonly encountered in multi-path channel environments, can be treated by employing appropriate techniques (e.g., spatial smoothing-based algorithm).

The paper is organized as follows: In Sect. 2, the system model is defined for both the conventional ULA and the hybrid structure receiver cases. In Sect. 3, the problem formulation for the full snapshot recovery is developed, along with the design issues of the analog combiner in the hybrid structure, investigating the issue for two different phaseshifter architectures. In Sect. 4, we show experimental results for the evaluation of the performance of the proposed scheme. Finally, Sect. 5 concludes the paper.

The following notations are used in this paper. Uppercase bold letters are matrices, lowercase bold letters are vectors, letters with a hat are estimations, $(\cdot)^{\mathrm{T}}$ denotes the transposition, $(\cdot)^{\mathrm{H}}$ denotes the complex conjugate transposition, $E[\cdot]$ means statistical expectation, $\operatorname{Tr}\{$. $\}$ denotes the trace of a matrix and $\mathbf{I}$ is the identity matrix.

\section{The system model}

Let us consider a ULA in which $L$ far-field, narrowband, bandpass signals impinge on. The $L$ signals arrive at the array from $L$ different directions. Moreover, the antenna array size $N$ is larger than the number of source signals, i.e., $N>L$ [20]. The spatial sampling depends on the wavelength of carrier frequency. In that case, to avoid spatial aliasing, the inter-element spacing should satisfy the condition $d=\frac{\lambda_{\mathrm{c}}}{2}=\frac{c}{2 f_{\mathrm{c}}}$, where $c$ is the propagation speed and $\lambda_{\mathrm{c}}, f_{\mathrm{c}}$ are the carrier wavelength and frequency. Furthermore, each source signal $i$ is associated with an AoA denoted as $\theta_{i}$, where $i=1,2, \ldots, L$ and 


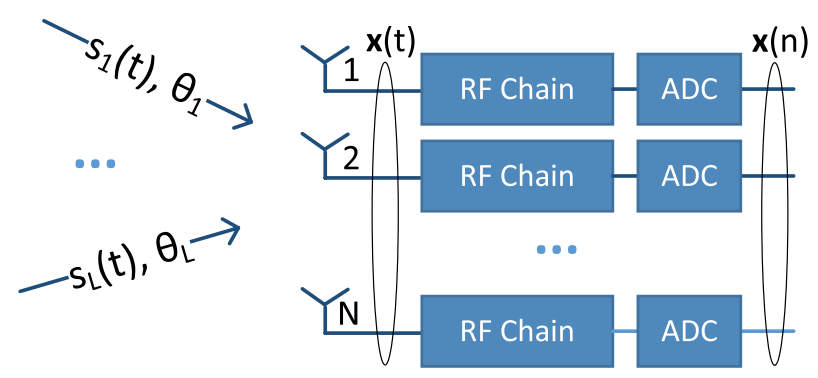

Fig. 1 The receiver in the conventional case

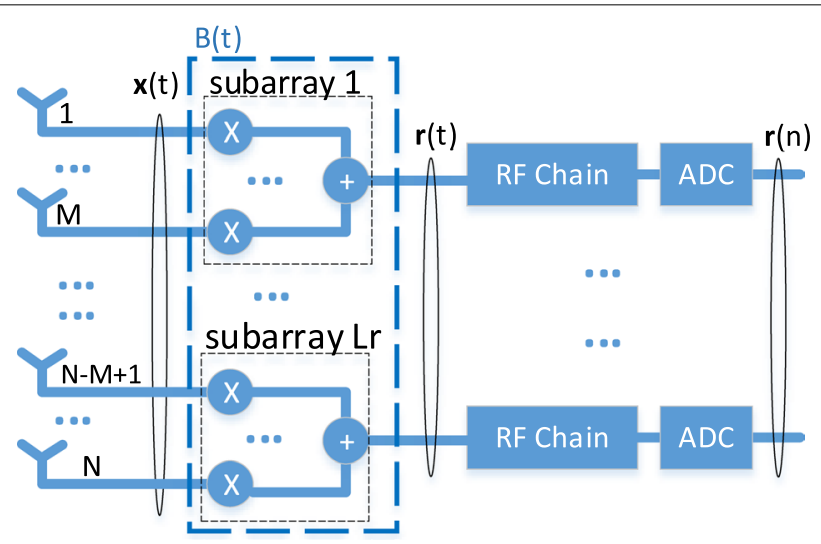

Fig. 2 The receiver in the partially connected hybrid case

$\theta_{i} \in[-\pi, \pi]$. We assume that AoAs do not change during snapshots collection time. Thus, the signal array on the time instant $t$ can be written as

$$
\mathbf{x}(t)=\mathbf{A}(\theta) \mathbf{s}(t)+\mathbf{w}(t),
$$

where $\mathbf{x}(t)=\left[x_{1}(t), x_{2}(t), \ldots, x_{N}(t)\right]$ is a $N \times 1$ vector with the signals received by the $N$ array elements, $\theta=\left[\theta_{1}(t), \theta_{2}(t), \ldots, \theta_{L}(t)\right]$ is an $L \times 1$ vector with the AoA's, $\mathbf{s}(t)=\left[s_{1}(t), s_{2}(t), \ldots, s_{L}(t)\right]^{\mathrm{T}}$ is an $L \times 1$ vector containing the source signals and $\mathbf{w}(t)=\left[w_{1}(t), w_{2}(t), \ldots, w_{N}(t)\right]$ is the $N \times 1$ noise vector. Finally, $\mathbf{A}(\theta)$ is the $N \times L$ array response matrix

$$
\mathbf{A}(\theta)=\left[\begin{array}{llll}
\mathbf{a}\left(\theta_{1}\right) & \mathbf{a}\left(\theta_{2}\right) & \cdots & \mathbf{a}\left(\theta_{L}\right)
\end{array}\right],
$$

where

$$
\mathbf{a}\left(\theta_{i}\right)=\frac{1}{\sqrt{N}}\left[\begin{array}{llll}
1 & \mathrm{e}^{j \frac{2 d \pi}{\lambda_{\mathrm{c}}} \sin \theta_{i}} & \cdots & \mathrm{e}^{j \frac{2(N-1) d \pi}{\lambda_{\mathrm{c}}} \sin \theta_{i}}
\end{array}\right]^{\mathrm{T}} .
$$

The sampled version of $\mathbf{x}(t)$ is usually utilized either by a conventional receiver, where each antenna element is connected to an RF chain (see Fig. 1), or by a hybrid structure receiver, where the signals of groups of antenna elements are, first, combined and the resulting signal is passed via an RF chain (see Figs. 2, 3). These two cases are analyzed in detail in the following subsections. 


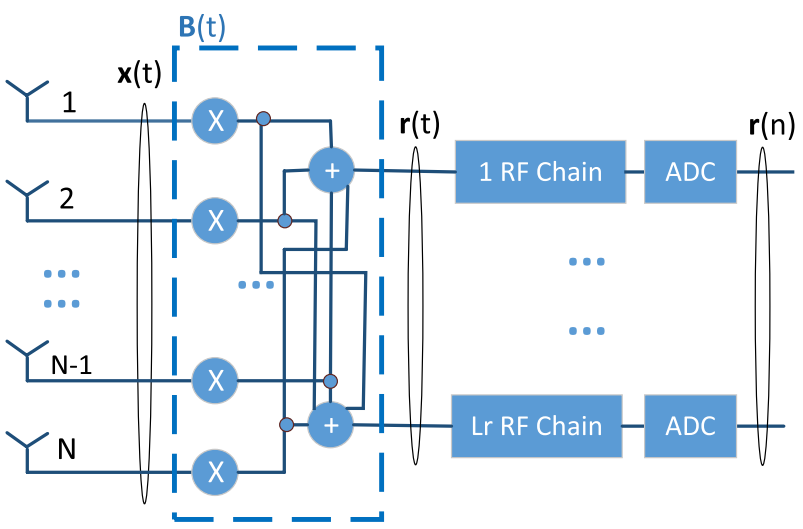

Fig. 3 The receiver in the fully connected hybrid case

\subsection{The classical ULA case}

Each received signal is processed by an individual RF chain, where, among other, is downconverted, and, then, is sampled by the corresponding analog-to-digital converter (ADC). At discrete-time $n$, the baseband signal, called full snapshot, is

$$
\mathbf{x}(n)=\mathbf{A}(\theta) \mathbf{s}(n)+\mathbf{w}(n),
$$

where $\mathbf{x}(n), \mathbf{w}(n) \in \mathrm{C}^{N}, \mathbf{s}(n) \in \mathrm{C}^{L}$ and $\mathbf{w}(n) \sim \mathrm{CN}\left(\mathbf{0}, \sigma^{2} \mathbf{I}\right)$, while $E\left[\mathbf{w}(n) \mathbf{w}^{\mathrm{H}}(k)\right]=\mathbf{0}$, for $n \neq k$ and $\mathbf{R}_{s}=E\left[\mathbf{s}(n) \mathbf{s}^{\mathrm{H}}(n)\right]$ the source signals autocorrelation matrix.

\subsection{The hybrid case}

We assume an antenna array with $N$ elements which can be organized into $L_{r}$ groups considering two widely used analog beamforming architectures, as shown in Figs. 2 and 3 . In the partially connected one, each group, called subarray, consists of $M$ antenna elements and connects to one RF chain, where $N=M L_{r}$. Furthermore, in the $l$ th group $\left(l=1,2, \ldots, L_{r}\right)$, the $i$ th received signal $(i=1,2, \ldots, M)$ is, first, processed by a phaseshifter by $\mathrm{e}^{j \phi_{l i}(t)}, \phi_{l i}(t) \in[-\pi, \pi]$, and, then, all of them are added up. In the fully connected one, all antenna elements form $L_{r}$ different antenna groups each of which connects to an RF chain. Similarly, each received signal at each antenna element is processed by a phase-shifter, and then, all of them are added up. In both architectures, the matrix $\mathbf{B}(t)$ of size $L_{r} \times N$, known as analog combiner in the relevant literature [1, 42], constitutes the analog part of the hybrid structure, as well as the involved operations (depicted in Figs. 2, 3). The receiver first processes the received signals using an $L_{r} \times N$ combiner, $\mathbf{B}(t)$, implemented using phase shifters such that $|\mathbf{B}(i ; j)|=1$. Therefore, the matrix $\mathbf{B}(t)$ maps the received signal $\mathbf{x}(t)$ to a reduced size signal vector $\mathbf{r}(t)$, namely $L_{r} \times 1$, where

$$
\mathbf{r}(t)=\mathbf{B}(t) \mathbf{x}(t) .
$$

Finally, as in the classical case, $\mathbf{r}(t)$ passes through the available RF chains and the corresponding ADCs and the resulting baseband signal can be written as

$$
\mathbf{r}(n)=\mathbf{B}(n) \mathbf{x}(n)
$$


or according to (4) in the following compact form,

$$
\mathbf{r}(n)=\mathbf{B}(n) \mathbf{A}(\theta) \mathbf{s}(n)+\mathbf{B}(n) \mathbf{w}(n) .
$$

Inspecting (6), the reconstruction of the full snapshot in (4) depends on the inversion of matrix $\mathbf{B}(n)$. However, it is non-square with $L_{r}<N$, i.e., it has more columns than rows, and thus, it is singular. In this case, the initial ULA snapshot $\mathbf{x}(n)$ cannot be restored. Hence, in the following section the adopted methodology for the full snapshot recovery is presented in detail.

\section{Recovering the full snapshot}

In this section, the problem of full snapshot reconstruction of the baseband signal in (4) is described. The adopted methodology consists of two parts: (1) the problem formulation for the full snapshot estimation and (2) the appropriate RF combiner design assuming both cases of time-varying and constant source signals for the hybrid architectures described previously.

\subsection{Description of the procedure for recovering the full snapshot}

Because of the hybrid architecture, as explained in the previous section, at discrete time $n$, we obtain a sub-snapshot $\mathbf{r}(n)$ of size $L_{r}$ instead of the desired one in (4). To reconstruct the full snapshot of (4), a number of sub-snapshots $\mathbf{r}(l)$ are collected and utilized. In the following, the description of this collection, which will permit the reconstruction of the full snapshot, will be presented. As it will be shown, the reconstruction accuracy depends, among other, on the variability of the source signals ${ }^{1}$ that impinge on the antenna array, which signals will be denoted as $\mathbf{s}_{v}(l)$ from now on. Both cases of time varying and constant source signals will be considered in the analysis of Sect. 3.2, where the optimal design of the respective combiners is discussed.

In more detail, let us assume that $T$ signals

$$
\mathbf{r}(l)=\mathbf{B}(l) \mathbf{A}(\theta) \mathbf{s}_{v}(l)+\mathbf{B}(l) \mathbf{w}(l)
$$

are collected at time instants $l=n, n+1, \ldots, n+T-1$, which will be referred to as sub-snapshots in the following. By concatenating the $T$ sub-snapshots into the $T L_{r} \times 1$ vector

$$
\overline{\mathbf{r}}(n)=\left[\begin{array}{c}
\mathbf{r}(n) \\
\mathbf{r}(n+1) \\
\vdots \\
\mathbf{r}(n+T-1)
\end{array}\right],
$$

the input-output relation can be formulated as

${ }^{1}$ In many radio communication standards, the involved signals exhibit fluctuations [43]. 


$$
\begin{aligned}
& \overline{\mathbf{r}}(n)= {\left[\begin{array}{c}
\mathbf{B}(n) \mathbf{A}(\theta) \mathbf{s}_{v}(n) \\
\mathbf{B}(n+1) \mathbf{A}(\theta) \mathbf{s}_{v}(n+1) \\
\vdots \\
\mathbf{B}(n+T-1) \mathbf{A}(\theta) \mathbf{s}_{v}(n+T-1)
\end{array}\right] } \\
& \mathbf{\mathbf { B } ( n ) \mathbf { w } ( n )} \\
&+\left[\begin{array}{c}
\mathbf{B}(n+1) \mathbf{w}(n+1) \\
\vdots \\
\mathbf{B}(n+T-1) \mathbf{w}(n+T-1)
\end{array}\right]
\end{aligned}
$$

or, in a more compact form,

$$
\overline{\mathbf{r}}(n)=\overline{\mathbf{B}}(n) \overline{\mathbf{A}}(\theta) \overline{\mathbf{s}}_{v}(n)+\overline{\mathbf{w}}(n),
$$

where

$$
\begin{aligned}
\overline{\mathbf{B}}(n) & =\left[\begin{array}{cccc}
\mathbf{B}(n) & \mathbf{0} & \ldots & \mathbf{0} \\
\mathbf{0} & \mathbf{B}(n+1) & \ldots & \mathbf{0} \\
\vdots & \vdots & \ddots & \vdots \\
\mathbf{0} & \mathbf{0} & \ldots & \mathbf{B}(n+T-1)
\end{array}\right], \\
\overline{\mathbf{A}}(\theta) & =\left[\begin{array}{cccc}
\mathbf{A}(\theta) & \mathbf{0} & \ldots & \mathbf{0} \\
\mathbf{0} & \mathbf{A}(\theta) & \ldots & \mathbf{0} \\
\vdots & \vdots & \ddots & \vdots \\
\mathbf{0} & \mathbf{0} & \ldots & \mathbf{A}(\theta)
\end{array}\right]
\end{aligned}
$$

and

$$
\overline{\mathbf{s}}_{v}(n)=\left[\begin{array}{llll}
\mathbf{s}_{v}^{\mathrm{T}}(n) & \mathbf{s}_{v}^{\mathrm{T}}(n+1) & \ldots & \mathbf{s}_{v}^{\mathrm{T}}(n+T-1)
\end{array}\right]^{\mathrm{T}} .
$$

In the following, the vectors $\mathbf{s}_{v}(l)$ involved in (14) are modeled as the sum of a constant signal $\mathbf{s}(n)$ and a non-constant signal $\mathbf{s}_{n c}(l)$, i.e., $\mathbf{s}_{v}(l)=\mathbf{s}(n)+\mathbf{s}_{n c}(l)$, for $l=n, n+1, \ldots, n+T-1$. Actually, it is practically infeasible to acquire constant source signals when operating at very high data rates (i.e., multiple Gbps depending on the mmWave carrier frequency), without oversampling. Therefore, here, the general case of non-constant signals is considered, as, the assumption of constant ones in a full snapshot period (usually encountered in the relevant literature [23]) can be treated as a special case, where $\mathbf{s}_{v}(l)=\mathbf{s}(n)$ for $l=n, n+1, \ldots, n+T-1$.

Specifically, the source signals vector in (14) can be rewritten as

$$
\overline{\mathbf{s}}_{v}(n) \equiv\left[\begin{array}{c}
\mathbf{s}(n)+\mathbf{s}_{n c}(n) \\
\mathbf{s}(n)+\mathbf{s}_{n c}(n+1) \\
\vdots \\
\mathbf{s}(n)+\mathbf{s}_{n c}(n+T-1)
\end{array}\right]
$$

Hence, by substituting (15) in (11) a collection of sub-snapshots of the hybrid array can be obtained as follows 


$$
\begin{aligned}
\overline{\mathbf{r}}(n)= & {\left[\begin{array}{c}
\mathbf{B}(n) \mathbf{A}(\theta) \mathbf{s}(n) \\
\mathbf{B}(n+1) \mathbf{A}(\theta) \mathbf{s}(n) \\
\vdots \\
\mathbf{B}(n+T-1) \mathbf{A}(\theta) \mathbf{s}(n)
\end{array}\right] } \\
& +\left[\begin{array}{c}
\mathbf{B}(n) \mathbf{A}(\theta) \mathbf{s}_{n c}(n) \\
\mathbf{B}(n+1) \mathbf{A}(\theta) \mathbf{s}_{n c}(n+1) \\
\vdots \\
\mathbf{B}(n+T-1) \mathbf{A}(\theta) \mathbf{s}_{n c}(n+T-1)
\end{array}\right] \\
& +\left[\begin{array}{c}
\mathbf{B}(n) \mathbf{w}(n) \\
\mathbf{B}(n+1) \mathbf{w}(n+1) \\
\vdots \\
\mathbf{B}(n+T-1) \mathbf{w}(n+T-1)
\end{array}\right]+
\end{aligned}
$$

or, in a more compact form,

$$
\overline{\mathbf{r}}(n)=\mathcal{B}(n) \mathbf{A}(\theta) \mathbf{s}(n)+\overline{\mathbf{B}}(n) \overline{\mathbf{A}}(\theta) \overline{\mathbf{s}}_{n c}(n)+\overline{\mathbf{w}}(n),
$$

where

$$
\mathcal{B}(n)=\left[\begin{array}{llll}
\mathbf{B}^{\mathrm{T}}(n) & \mathbf{B}^{\mathrm{T}}(n+1) & \ldots & \mathbf{B}^{\mathrm{T}}(n+T-1)
\end{array}\right]^{\mathrm{T}}
$$

and

$$
\overline{\mathbf{s}}_{n c}(n)=\left[\begin{array}{llll}
\mathbf{s}_{n c}^{\mathrm{T}}(n) & \mathbf{s}_{n c}^{\mathrm{T}}(n+1) & \ldots & \mathbf{s}_{n c}^{\mathrm{T}}(n+T-1)
\end{array}\right]^{\mathrm{T}} .
$$

The value of parameter $T$ (i.e., the number of sub-snapshots) is selected such that the $T L_{r} \times N$ matrix $\mathcal{B}(n)$ is invertible, thus, $T L_{r} \geq N$ should hold. To ensure low sub-snapshots collection overhead the minimum value $T=\frac{N}{L_{r}}$ is chosen, where the number of antenna elements $N$ and the number of RF chains $L_{r}$ are considered to be powers of two, i.e., $N=2^{i}$ and $L_{r}=2^{j}$, where $i, j$ are integers. By applying different matrices $\mathbf{B}(l)$ for each $l$, linear independent $\mathbf{r}(l)$ 's are collected. Due to the fact that $\mathcal{B}(n)$ is designed to be rectangular and full rank, its pseudo-inverse matrix $\mathcal{B}^{\dagger}(n)$ is reduced to $\mathcal{B}^{-1}(n)$. In the following, from (17) an estimate $\hat{\mathbf{x}}(n)$ of the full snapshot $\mathbf{x}(n)$ can be acquired as

$$
\hat{\mathbf{x}}(n)=\mathcal{B}^{-1}(n) \overline{\mathbf{r}}(n) .
$$

Hence, from (20) and as outlined in Algorithm 1, we can fully recover the full snapshot as

$$
\begin{aligned}
\hat{\mathbf{x}}(n)= & \mathbf{A}(\theta) \mathbf{s}(n)+\mathcal{B}^{-1}(n) \overline{\mathbf{w}}(n) \\
& +\mathcal{B}^{-1}(n) \overline{\mathbf{B}}(n) \overline{\mathbf{A}}(\theta) \overline{\mathbf{s}}_{n c}(n) .
\end{aligned}
$$

According to (21), the $\hat{\mathbf{x}}(n)$ is hindered by a term associated with the additive noise and an additional term which depends on the time variability of the received source signals. As it will be observed in the following, the variability in the source signals is treated as a noise term. Hence, in this case, the total noise term is

$$
\mathbf{w}^{\prime \prime}(n)=\mathcal{B}^{-1}(n) \overline{\mathbf{w}}(n)+\mathcal{B}^{-1}(n) \overline{\mathbf{B}}(n) \overline{\mathbf{A}}(\theta) \overline{\mathbf{s}}_{n c}(n) .
$$


Considering now the special case of constant signals, namely $\mathbf{s}_{v}(l)=\mathbf{s}(n)$, for $l=n, n+1, \ldots, n+T-1, \overline{\mathbf{s}}_{v}(n)$ is simplified to

$$
\overline{\mathbf{s}}_{v}(n)=\left[\begin{array}{c}
\mathbf{s}(n) \\
\mathbf{s}(n) \\
\vdots \\
\mathbf{s}(n)
\end{array}\right] .
$$

Hence, $\overline{\mathbf{r}}(n)$ can be written as

$$
\overline{\mathbf{r}}(n)=\mathcal{B}(n) \mathbf{A}(\theta) \mathbf{s}(n)+\overline{\mathbf{w}}(n)
$$

and the restored full snapshot in this case is given as

$$
\hat{\mathbf{x}}(n)=\mathbf{A}(\theta) \mathbf{s}(n)+\mathcal{B}^{-1}(n) \overline{\mathbf{w}}(n),
$$

where the total noise term $\mathbf{w}^{\prime \prime}(n)$ in (22) is reduced to

$$
\mathbf{w}^{\prime \prime}(n)=\mathcal{B}^{-1}(n) \overline{\mathbf{w}}(n) .
$$

From (21) and (25), it is evident that the matrix $\mathcal{B}(n)$ has an impact on the power of the noise terms thus affecting the subsequent utilization of the recovered signals, i.e., in AoA estimation. Hence, the matrix $\mathcal{B}(n)$ should be determined appropriately so that it invertible and well conditioned and, at the same time, it does not increase the power of the noise term. Such a discussion and the relevant analysis are presented in the following section.

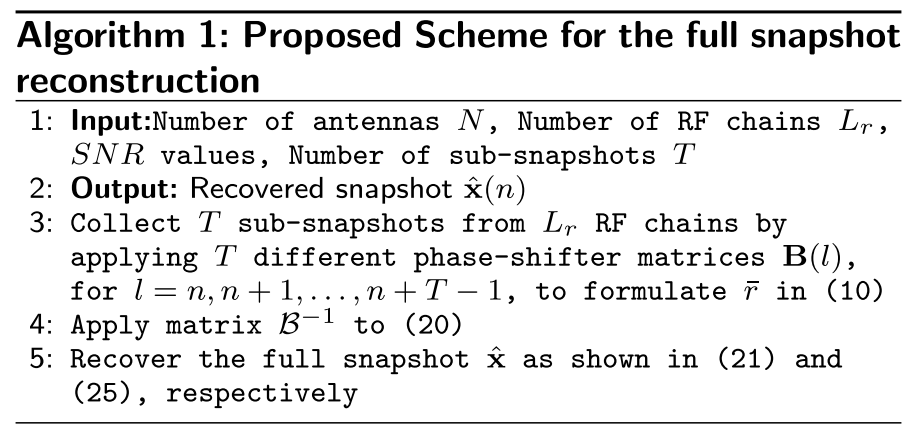

\subsection{Problem formulation for the RF combiner design}

In this section, the analysis focuses on the appropriate design of the RF combiner $\mathcal{B}(n)$ matrix, i.e., the analog part of the hybrid structure for the minimum value of $T=\frac{N}{L_{r}}$.

The problem of interest in this paper is to design the $\mathcal{B}(n)$, i.e., the analog part of the hybrid structure, such that the STNR, after the application of the preprocessing scheme, to be maximized. The STNR is defined as

$$
\operatorname{STNR}=\frac{\operatorname{Tr}\{\mathbf{C}\}}{\operatorname{Tr}\left\{\mathbf{C}_{\mathbf{w}^{\prime \prime}}\right\}},
$$

where the power of the signal component of ULA snapshot $\mathbf{x}(n)$ in (4), defined as 


$$
\begin{aligned}
\operatorname{Tr}\{\mathbf{C}\} & =\operatorname{Tr}\left\{\mathbf{A}(\theta) E\left[\mathbf{s}(n) \mathbf{s}^{\mathrm{H}}(n)\right] \mathbf{A}(\theta)^{\mathrm{H}}\right\} \\
& =\operatorname{Tr}\left\{\mathbf{A}(\theta) \mathbf{R}_{s} \mathbf{A}(\theta)^{\mathrm{H}}\right\},
\end{aligned}
$$

is kept constant and identical to the one in the reconstructed full snapshot. Hence, the STNR maximization is equivalent to the minimization of the total noise power $\operatorname{Tr}\left\{\mathbf{C}_{\mathbf{w}^{\prime \prime}}\right\}$ which entails that the total noise term $\mathbf{w}^{\prime \prime}(n)=\mathcal{B}^{-1}(n) \overline{\mathbf{w}}(n)+\mathcal{B}^{-1}(n) \overline{\mathbf{B}}(n) \overline{\mathbf{A}}(\theta) \overline{\mathbf{s}}_{n c}(n)$ in (22) and the noise term in (4) have equivalent statistical properties (e.g., as to covariance matrix). It is noted, here, that in the special case of constant signals, $\mathbf{w}^{\prime \prime}(n)$ is equal to $\mathcal{B}^{-1}(n) \overline{\mathbf{w}}(n)$. The desired minimization problem, which lies in the minimization of the power of the total noise term $\mathbf{w}^{\prime \prime}(n)$, is written as,

$$
\min _{\mathcal{B}(n)} \operatorname{Tr}\left\{\mathbf{C}_{\mathbf{w}^{\prime \prime}}\right\} \text {, s.t. } \mathcal{B}(n) \in \mathrm{S},
$$

where $\mathbf{C}_{\mathbf{w}^{\prime \prime}}=E\left\{\mathbf{w}^{\prime \prime}(n)\left(\mathbf{w}^{\prime \prime}(n)\right)^{\mathrm{H}}\right\}$ is the covariance matrix of the noise term in (21) and $S$ denotes the feasibility set of $\mathcal{B}(n)$, namely all matrices with elements being equal to exponentials that denote the phase-shifting operation of the hybrid architecture (either fully or partially connected). In particular, the set S consists of all $N \times N$ matrices, of the form

$$
\mathcal{B}(n)=\left[\begin{array}{c}
\mathbf{B}(n) \\
\mathbf{B}(n+1) \\
\vdots \\
\mathbf{B}(n+T-1)
\end{array}\right] .
$$

The form of sub-matrices $\mathbf{B}(n)$ in (30) captures both the structure and the operations, described in Sect. 3.1, and will be elaborated in the following. If the hybrid architecture is organized into non-overlapping subarrays as depicted in Fig. 2, in (30) the sub-matrices are of the form

$$
\mathbf{B}(l)=\left[\begin{array}{cccc}
\mathbf{b}_{l 1}^{\mathrm{T}} & \mathbf{0} & \ldots & \mathbf{0} \\
\mathbf{0} & \mathbf{b}_{l 2}^{\mathrm{T}} & \ldots & \mathbf{0} \\
\vdots & \vdots & \ddots & \vdots \\
\mathbf{0} & \mathbf{0} & \ldots & \mathbf{b}_{l L_{r}}^{\mathrm{T}}
\end{array}\right]
$$

where $\mathbf{b}_{l j}$ is an $M \times 1$ vector with the phase-shifters' values to be used by the $j$ th subarray at time $l=n, n+1, \ldots, n+T-1$. Assuming a fully connected architecture, as in Fig. 3, the sub-matrices in (30) are of the form

$$
\mathbf{B}(l)=\left[\begin{array}{c}
\mathbf{b}_{l 1}^{\mathrm{T}} \\
\mathbf{b}_{l 2}^{\mathrm{T}} \\
\vdots \\
\mathbf{b}_{l L_{r}}^{\mathrm{T}}
\end{array}\right]
$$

and $\mathbf{b}_{l j}$ is an $N \times 1$ vector with the exponentials to be used by the $j$ th subarray at time $l=n, n+1, \ldots, n+T-1$. Here, it should be noted that the same notation $\mathbf{b}_{l j}$ for two 
different sized vectors is utilized so as to keep the symbols simple and the dimension is implied by the context.

In the following, the minimization problem of (29) will be solved for the two architectures (Figs. 2, 3) under study and the two signal cases (i.e., for constant and non-constant signals described in (23) and (15), respectively). As it will be shown later, when constant signals are considered, the problem can be solved optimally, whereas for the non-constant signals the selection of the appropriate design becomes a more intriguing task.

\subsubsection{Constant source signals}

First, the special case of constant source signals will be considered. Under this case, the noise term $\mathbf{w}^{\prime \prime}(n)$ is equal to $\mathcal{B}^{-1}(n) \overline{\mathbf{w}}(n)$, as shown in (25), and the covariance matrix can be written as $\mathbf{C}_{\mathbf{w}^{\prime \prime}}=E\left\{\mathcal{B}^{-1}(n) \overline{\mathbf{w}}(n)\left(\mathcal{B}^{-1}(n) \overline{\mathbf{w}}(n)\right)^{\mathrm{H}}\right\}$. In order to solve the problem in (29), first, $\mathbf{C}_{\mathbf{w}^{\prime \prime}}$ is written in the following equivalent form,

$$
\mathbf{C}_{\mathbf{w}^{\prime \prime}}=\mathcal{B}^{-1}(n) E\left\{\overline{\mathbf{w}}(n)(\overline{\mathbf{w}}(n))^{\mathrm{H}}\right\}\left(\mathcal{B}^{-1}(n)\right)^{\mathrm{H}} .
$$

In the following, using (33), (29) will be solved for the two hybrid architectures. First, the partially connected case will be treated.

In the partially connected architecture, it can be proved, by employing (30), (31), that $E\left\{\overline{\mathbf{w}}(n)(\overline{\mathbf{w}}(n))^{\mathrm{H}}\right\}=\sigma^{2} M \mathbf{I}_{N \times N}$, and, thus, $\mathbf{C}_{\mathbf{w}^{\prime \prime}}$ is written as

$$
\mathbf{C}_{\mathbf{w}^{\prime \prime}}=\sigma^{2} M\left(\mathcal{B}^{\mathrm{H}}(n) \mathcal{B}(n)\right)^{-1}
$$

Moreover, the following inequality will be used for determining a lower bound for the cost function in (29),

$$
\operatorname{Tr}\left\{\mathbf{A}^{-1}\right\} \geq \sum_{i} \frac{1}{[\mathbf{A}]_{i i}}
$$

where $[\mathbf{A}]_{i i}$ is the $i$ th diagonal element of $\mathbf{A}$ and the equality holds when $\mathbf{A}$ is diagonal [44]. By substituting (34) in (29) and applying (35), the following lower bound can be derived,

$$
\operatorname{Tr}\left\{\mathbf{C}_{\mathbf{w}^{\prime \prime}}\right\} \geq \sigma^{2} M \sum_{i=1}^{N} \frac{1}{\left[\mathcal{B}^{\mathrm{H}}(n) \mathcal{B}(n)\right]_{i i}}=\sigma^{2} N .
$$

Inspecting (36), the lower bound is independent of the unknown $\mathbf{B}(n)$ we are looking for. Therefore, any $\mathcal{B}(n) \in \mathrm{S}$ can be considered as the optimal solution in (29). In our case, an optimal choice of $\mathbf{B}(n)$ is the one for which the following condition is fulfilled

$$
\mathcal{B}^{\mathrm{H}}(n) \mathcal{B}(n)=M \mathbf{I},
$$

which subsequently makes the covariance matrix $\mathbf{C}_{\mathbf{w}^{\prime \prime}}$ diagonal and equal to the one of the noise term in (4), i.e., $\mathbf{C}_{\mathbf{w}^{\prime \prime}}=\sigma^{2} \mathbf{I}$ (as observed by 34). In the following, such an optimal, in this setting, matrix will be described.

In order to determine a suitable $\mathcal{B}(n) \in \mathrm{S}$, first, $\mathcal{B}^{\mathrm{H}}(n) \mathcal{B}(n)$ is written in the following more convenient form 


$$
\mathcal{B}^{\mathrm{H}}(n) \mathcal{B}(n)=\mathcal{B}^{\mathrm{H}}(n) \mathbf{P}^{\mathrm{T}} \mathbf{P} \mathcal{B}(n),
$$

where $\mathbf{P}$ is an $N \times N$ permutation matrix that changes the position of the rows of $\mathcal{B}(n)$ in the following manner. The first row of each $\mathbf{B}(l)$ goes at the top (see (30), (31)). Then, the second row of each $\mathbf{B}(l)$ follows and so on. This matrix can be created from the identity matrix if its rows are moved similarly. The matrix $\mathbf{P} \mathcal{B}(n)$ can be written as

$$
\mathbf{P} \mathcal{B}(n)=\left[\begin{array}{cccc}
\mathbf{F}_{1} & \mathbf{0} & \ldots & \mathbf{0} \\
\mathbf{0} & \mathbf{F}_{2} & \ldots & \mathbf{0} \\
\vdots & \vdots & \ddots & \vdots \\
\mathbf{0} & \mathbf{0} & \ldots & \mathbf{F}_{L_{r}}
\end{array}\right]
$$

where $\mathbf{F}_{i}, i=1,2, \ldots, L_{r}$, is an $M \times M$ matrix with its $l$ th row equal to $\mathbf{b}_{l i}^{\mathrm{T}}$, $l=n, n+1, \ldots, n+M-1$.

After substituting (39) in $(38), \mathcal{B}^{\mathrm{H}}(n) \mathcal{B}(n)$ is a block diagonal matrix, as shown in (40), with the $i$ th diagonal element being $\mathbf{F}_{i}^{\mathrm{H}} \mathbf{F}_{i}, i=1,2, \ldots, L_{r}$.

$$
\mathcal{B}^{\mathrm{H}}(n) \mathcal{B}(n)=\left[\begin{array}{cccc}
\mathbf{F}_{1}^{\mathrm{H}} \mathbf{F}_{1} & \mathbf{0} & \ldots & \mathbf{0} \\
\mathbf{0} & \mathbf{F}_{2}^{\mathrm{H}} \mathbf{F}_{2} & \ldots & \mathbf{0} \\
\vdots & \vdots & \ddots & \vdots \\
\mathbf{0} & \mathbf{0} & \ldots & \mathbf{F}_{L_{r}}^{\mathrm{H}} \mathbf{F}_{L_{r}}
\end{array}\right]
$$

Thus, the desired condition depicted by (37) can be fulfilled if equivalently $\mathbf{F}_{i}^{\mathrm{H}} \mathbf{F}_{i}=M \mathbf{I}$.

An appropriate choice for the $\mathbf{F}_{i}$ 's, $i=1,2, \ldots, L_{r}$, could be based on the Fourier matrix $\mathbf{F}_{N_{\times} N}$, whose $(p, q)$ th element is given as $[\mathbf{F}]_{p, q}=\mathrm{e}^{-j 2 \pi p q / N}$. Then, only the first $M$ columns are kept and the resulting matrix is denoted as $\mathbf{F}_{N \times M}$. Finally, rows of $\mathbf{F}_{i}$, $i=1,2, \ldots, L_{r}$, are set equal to the rows $i, L_{r}+i, 2 L_{r}+i, \ldots$ of $\mathbf{F}_{N \times M}$. These matrices fulfill the desired condition, namely $\mathbf{F}_{i}^{\mathrm{H}} \mathbf{F}_{i}=M \mathbf{I}$. Hence, by utilizing these matrices, the phase shifters of the receiver in the partially connected, hybrid case can be determined. It is noted, here, that the $\mathbf{F}_{i}$ 's are actually the beamforming matrices used by $\mathrm{H}$-MUSIC in [23] in order to obtain the overall received vector, assuming $T=M$, e.g., rectangular beamformers. It is worth mentioning that H-MUSIC can work with nonrectangular beamformers $\mathbf{F}_{i}$ 's, i.e., with $T<M$, thus, with smaller size sub-snapshots and snapshots whose size will be less than the so-called full snapshot.

Here, the case of the fully connected architecture is investigated. In order to solve the problem in (29) by employing (32), it can be shown that $E\left\{\overline{\mathbf{w}}(n)(\overline{\mathbf{w}}(n))^{\mathrm{H}}\right\}=\sigma^{2} \mathcal{D}(n)$, where matrix $\mathcal{D}(n)$ is block diagonal with diagonal elements of the form $\mathbf{B}(l) \mathbf{B}^{H}(l)$ for $l=n, n+1, \ldots, n+T-1$. Using above, the trace of $\mathbf{C}_{\mathbf{w}^{\prime \prime}}$ in (33) is written in the following equivalent form,

$$
\begin{aligned}
\operatorname{Tr}\left\{\mathbf{C}_{\mathbf{w}^{\prime \prime}}\right\} & =\operatorname{Tr}\left\{\sigma^{2} \mathcal{B}^{-1}(n) \mathcal{D}(n)\left(\mathcal{B}^{-1}(n)\right)^{H}\right\} \\
& =\operatorname{Tr}\left\{\sigma^{2} \mathcal{D}(n)\left(\mathcal{B}^{-1}(n)\right)^{H} \mathcal{B}^{-1}(n)\right\} \\
& =\operatorname{Tr}\left\{\sigma^{2} \mathcal{D}(n)\left(\mathcal{B}(n) \mathcal{B}^{\mathrm{H}}(n)\right)^{-1}\right\} \\
& =\operatorname{Tr}\left\{\sigma^{2}\left(\mathcal{B}(n) \mathcal{B}^{\mathrm{H}}(n) \mathcal{D}^{-1}(n)\right)^{-1}\right\} .
\end{aligned}
$$

By substituting (41) in (29) and applying (35), the following lower bound can be derived, 


$$
\operatorname{Tr}\left\{\mathbf{C}_{\mathbf{w}^{\prime \prime}}\right\} \geq \sigma^{2} \sum_{i=1}^{N} \frac{1}{\left[\mathcal{B}(n) \mathcal{B}^{\mathrm{H}}(n) \mathcal{D}^{-1}(n)\right]_{i i}}=\sigma^{2} N
$$

where, for deriving the last equality, $\left[\mathcal{B}(n) \mathcal{B}^{\mathrm{H}}(n) \mathcal{D}^{-1}(n)\right]_{i i}=1, \forall i$, is used, which can be proved by employing (30), (32) and observing the diagonal elements of the result.

Observing (42), any matrix could be utilized for deriving the lower bound. However, in this case, the matrix $\mathcal{B}(n)$ is designed based on the Fourier matrix $\mathbf{F}_{N \times N}$, as this one has the properties of S. Then, submatrices of $\mathbf{F}$ of size $L_{r} \times N$ can be used for the design of the $\mathbf{B}(l)$ s for $l=n, n+1, \ldots, n+T-1$ that comprise the matrix $\mathcal{B}(n)$ in (30). Hence, by utilizing these matrices the phase shifters of the receiver in the fully connected, hybrid case can be determined, as, in this case, the minimum value in (36) is satisfied.

\subsubsection{Non-constant source signals}

In this section, the case of non-constant source signals will be elaborated. In order to solve the problem in (29), the covariance matrix, $\mathbf{C}_{\mathbf{w}^{\prime \prime}}$, for the noise term $\mathbf{w}^{\prime \prime}(n)=\mathcal{B}^{-1}(n) \overline{\mathbf{w}}(n)+\mathcal{B}^{-1}(n) \overline{\mathbf{B}}(n) \overline{\mathbf{A}}(\theta) \overline{\mathbf{s}}_{n c}(n)$ can be written as

$$
\begin{aligned}
\mathbf{C}_{\mathbf{w}^{\prime \prime}}= & E\left\{\mathbf{w}^{\prime \prime}(n)\left(\mathbf{w}^{\prime \prime}(n)\right)^{\mathrm{H}}\right\} \\
= & \sigma^{2} \mathcal{B}^{-1}(n) \overline{\mathbf{B}}(n) \overline{\mathbf{B}}^{\mathrm{H}}(n)\left(\mathcal{B}^{-1}(n)\right)^{H} \\
& +\mathcal{B}^{-1}(n) \overline{\mathbf{B}}(n) \overline{\mathbf{A}}(\theta) \mathbf{R}_{\overline{\mathbf{s}}_{n c}} \overline{\mathbf{A}}^{\mathrm{H}}(\theta) \overline{\mathbf{B}}^{\mathrm{H}}(n)\left(\mathcal{B}^{-1}(n)\right)^{H} \\
= & \mathcal{B}^{-1}(n)\left(\sigma^{2} \overline{\mathbf{B}}(n) \overline{\mathbf{B}}^{\mathrm{H}}(n)\right. \\
& \left.+\overline{\mathbf{B}}(n) \overline{\mathbf{A}}(\theta) \mathbf{R}_{\overline{\mathbf{s}}_{n c}} \overline{\mathbf{A}}^{\mathrm{H}}(\theta) \overline{\mathbf{B}}^{\mathrm{H}}(n)\right)\left(\mathcal{B}^{-1}(n)\right)^{H} .
\end{aligned}
$$

By substituting (43) into the cost function of (29), the following equation can be written as

$$
\operatorname{Tr}\left\{\mathbf{C}_{\mathbf{w}^{\prime \prime}}\right\}=\operatorname{Tr}\left\{\mathcal{B}^{-1}(n) \mathbf{U}\left(\mathcal{B}^{-1}(n)\right)^{\mathrm{H}}\right\},
$$

where $\mathbf{U}=\sigma^{2} \overline{\mathbf{B}}(n) \overline{\mathbf{B}}^{\mathrm{H}}(n)+\overline{\mathbf{B}}(n) \overline{\mathbf{A}}(\theta) \mathbf{R}_{\overline{\mathbf{s}}_{n c}} \overline{\mathbf{A}}^{\mathrm{H}}(\theta) \overline{\mathbf{B}}^{\mathrm{H}}(n)$ and $\mathbf{R}_{\overline{\mathbf{s}}_{n c}}$ the non-constant signals correlation matrix of size $T L \times T L$.

For the full snapshot reconstruction the preprocessing matrix $\mathcal{B}(n)$ in (30) should not change the noise power, thus, the set of solutions $S$ is further restricted into a new set that consists of only unitary matrices, i.e., $\mathcal{B}^{-1}(n)=\mathcal{B}^{\mathrm{H}}(n)$. Hence, the trace of (44) can be further simplified as

$$
\begin{aligned}
\operatorname{Tr}\left\{\mathbf{C}_{\mathbf{w}^{\prime \prime}}\right\}= & \operatorname{Tr}\left\{\mathcal{B}^{\mathrm{H}}(n) \mathbf{U} \mathcal{B}(n)\right\} \\
= & \operatorname{Tr}\left\{\mathbf{U} \mathcal{B}(n) \mathcal{B}^{\mathrm{H}}(n)\right\} \\
= & \operatorname{Tr}\{\mathbf{U}\} \\
= & \operatorname{Tr}\left\{\sigma^{2} \overline{\mathbf{B}}(n) \overline{\mathbf{B}}^{\mathrm{H}}(n)\right. \\
& \left.\left.+\overline{\mathbf{B}}(n) \overline{\mathbf{A}}(\theta) \mathbf{R}_{\overline{\mathbf{s}}_{n c}} \overline{\mathbf{A}}^{\mathrm{H}}(\theta)\right) \overline{\mathbf{B}}^{\mathrm{H}}(n)\right\} \\
= & \operatorname{Tr}\left\{\overline{\mathbf{B}}(n)\left(\sigma^{2} \mathbf{I}_{T N}+\overline{\mathbf{A}}(\theta) \mathbf{R}_{\overline{\mathbf{s}}_{n c}} \overline{\mathbf{A}}^{\mathrm{H}}(\theta)\right) \overline{\mathbf{B}}^{\mathrm{H}}(n)\right\} \\
= & \operatorname{Tr}\left\{\overline{\mathbf{B}}(n) \mathbf{V} \overline{\mathbf{B}}^{\mathrm{H}}(n)\right\},
\end{aligned}
$$


where $\mathbf{V}=\sigma^{2} \mathbf{I}_{T N}+\overline{\mathbf{A}}(\theta) \mathbf{R}_{\overline{\mathbf{s}}_{n c}} \overline{\mathbf{A}}^{\mathrm{H}}(\theta)$. Next, according to [44], the lower bound of (29), when the cost-function has the form of (45), can be written as

$$
\operatorname{Tr}\left\{\mathbf{C}_{\mathbf{w}^{\prime \prime}}\right\} \geq \sum_{i=1}^{N} \lambda_{i}(\mathbf{V}),
$$

where the $\lambda_{i}$ s' for $i=1,2, \ldots, T N$, are the eigenvalues of the matrix $\mathbf{V}$.

Irrespective of the hybrid architecture, the minimum value in (46) is attained when the matrix $\overline{\mathbf{B}}(n)$ equals the matrix of eigenvectors of $\mathbf{V}$ that correspond to the $N$ smallest eigenvalues, sorted in an ascending order. It is noted, here, that $\overline{\mathbf{A}}(\theta) \mathbf{R}_{\overline{\mathbf{s}}_{n c}} \overline{\mathbf{A}}^{\mathrm{H}}(\theta)$ of size $T N \times T N$ is a matrix of rank equal to $T L$. If the number of antenna elements $N$ and the number of sources $L$ satisfy the condition $T N-T L>N$ this entails that the $N$ smallest eigenvalues are equal to $\sigma^{2}$, and so the minimum value in (46) can be written as $\min \operatorname{Tr}\left\{\mathbf{C}_{\mathbf{w}^{\prime \prime}}\right\}=\sigma^{2} N$.

The solution in (46) that minimizes the problem in (29), does not have the desired phase shifters' matrix structure because of the term $\overline{\mathbf{A}}(\theta) \mathbf{R}_{\overline{\mathbf{s}}_{n c}} \overline{\mathbf{A}}^{\mathrm{H}}(\theta)$ in matrix $\mathbf{V}$, which depends on the unknown AoAs.

Under the assumption of a low SNR regime, in the involved cost function in (45), the dominant term would be the one related to the additive noise, especially when the time variations of the source signals are not strong enough. Hence, in this case, (45) can be approximated by $\operatorname{Tr}\left\{\sigma^{2} \overline{\mathbf{B}}(n) \overline{\mathbf{B}}^{\mathrm{H}}(n)\right\}$. To design the $\mathcal{B}(n)$, for either partially connected or fully connected architecture, a similar process as the one followed in case of constant signals can be employed. As it has been already proved in the previous section, the Fourier matrix is an appropriate choice for the design of matrix $\mathcal{B}(n)$ for both hybrid architectures, as this one has the properties of $S$ (i.e., all matrices with elements being equal to exponentials that denote the phase-shifting operation of the hybrid architecture) and satisfies the minimum value $\sigma^{2} N$ in (46).

Finally, in the medium to high SNR regime, the dominant term in matrix $\mathbf{V}$ is $\overline{\mathbf{A}}(\theta) \mathbf{R}_{\mathbf{s}_{n c}} \overline{\mathbf{A}}^{\mathrm{H}}(\theta)$. Hence, the solution to the minimization problem in (29) is a matrix which actually depends on the unknown AoAs, which constitutes this case intractable, and the statistical properties of the time varying signals $\mathbf{s}_{n c}(l)$. Since, in this case, the solution to the minimization problem under study is related to the unknown AoAs, any predefined matrix is expected to lead to a floor at the cost function of (45). This floor depends on how powerful the time-varying nature of the source signals are (namely, on the values of the covariance matrix $\mathbf{R}_{\overline{\mathbf{s}}_{n c}}$, if one considers the model that is adopted here). Since the modeling of $\mathbf{R}_{\overline{\mathbf{s}}_{n c}}$ is out of the scope of this paper a simple model for the time-varying signals $\mathbf{s}_{n c}(l)$ can be adopted. Specifically, they can be modeled as discrete random variables of Gaussian distribution assuming that their values are statistically independent of equal power, with known first and second order statistics (i.e., $\mathbf{s}_{n c}(l) \sim \mathcal{C} N\left(\mathbf{0}, \sigma_{n c}^{2} \mathbf{I}\right)$ ). Under this model, the noise power behavior in (45) is verified in the simulations which are presented in the next section.

\section{Results and discussion}

In this section, simulations are carried out to demonstrate the performance of the proposed scheme and verify its behavior under the various cases that have been studied previously. To be more specific, the recoverability of the full snapshot is considered for 
the problem of AoA estimation in 1D mmWave massive MIMO systems by employing MUSIC. The combination of the proposed full snapshot reconstruction scheme with the classical MUSIC algorithm is called hereafter PRE+MUSIC.

In the following, we assume that there are $L=4$ signal sources. The constant part $\mathbf{s}(n)$ of the source signals is assumed to be an i.i.d. sequence of Quadrature Phase Shift Keying (QPSK) symbols. These signals impinge on the array with AoAs equal to $12.3^{\circ}, 28.1^{\circ}$, $54.6^{\circ}, 62.8^{\circ}$, respectively. The carrier frequency $f_{\mathrm{c}}$ is selected in the mmWave band and it is set to $30 \mathrm{GHz}$, which corresponds to a wavelength $\lambda$ equals to $1 \mathrm{~cm}$ and antenna spacing $0.5 \mathrm{~cm}$. An $N=32$ elements antenna array is deployed in receiver side which, in the hybrid case, is grouped into $L_{r}=8 \mathrm{RF}$ chains. In the following, three experiments will be presented.

In the first experiment, we demonstrate how well the theoretical analysis, presented in Sect. 3.2, predict the performance of the proposed scheme for the full snapshot reconstruction and validate the agreement between the computed theoretical minimum of the total noise power $\operatorname{Tr}\left\{\mathbf{C}_{\mathbf{w}^{\prime \prime}}\right\}$, under the optimal DFT-based phase-shift combiner matrix in the case of constant signals. Moreover, the performance improvement of the proposed solution over the random phase-shift combiner matrix is also demonstrated. In the following experiments we investigate the performance of the proposed full snapshot reconstruction scheme in the context of AoA estimation.

\subsection{Noise power in reconstructed snapshots}

Let recall that, since the signal component power remains constant, the STNR values in (27) depend only on the corresponding total noise power $\operatorname{Tr}\left\{\mathbf{C}_{\mathbf{w}^{\prime \prime}}\right\}$, which, in the case of non-constant sources is dependent on the additive noise power $\sigma^{2}$ plus a term which depends on the non-constant signals power $\sigma_{n c}^{2}$, treated as noise term, as well. In the special case of constant sources, the total noise term consists only of the additive noise. Hence, the power of the noise component $\left(\mathbf{w}^{\prime \prime}(n)\right)$ in the reconstructed snapshot [see (22) for non-constant sources or (26) for constant ones] is depicted in Fig. 4, versus the additive noise power $\sigma^{2}$, for $\sigma_{n c}^{2}=\{0,0.01,0.1\}$ (denoting the power of $\mathbf{s}_{n c}$ ), when the Fourier matrix for the phase shifters is utilized, for the fully connected architecture. It is worth to mention that the behavior of the cost function remains the same if, instead of the fully connected architecture, the partially connected architecture is adopted in each case. Furthermore, the theoretical minimum value in (36), (42) is also depicted in the same figure when the Fourier matrix is considered. Indeed, when $\sigma_{n c}^{2}=0$ the Fourier matrix achieves the minimum value and constitutes an optimal choice. As discussed in Sect. 3.2, the proposed phase shifters for both architectures, in the case when $\sigma_{n c}^{2} \neq 0$, follow the minimum value at the low SNR regime. Additionally, as $\sigma_{n c}^{2}$ increases, the floor of the cost function starts appearing in smaller values of SNR. Also, inspecting Fig. 4, when considering random phase-shifters combiner matrices $\mathcal{B}(n)$ at the cost function in (44), it is observed that the theoretical minimum value of $\sigma^{2} N$ cannot be achieved because the inequality here is strict. Moreover, the use of random phase-shifters combiner matrix, shifted the total noise power values, however, the form of figures remained the same. Note that, the resulted figures in case of random phases combiner illustrate the average values of $\operatorname{Tr}\left\{\mathbf{C}_{\mathbf{w}^{\prime \prime}}\right\}$ for 500 different random combiners. 


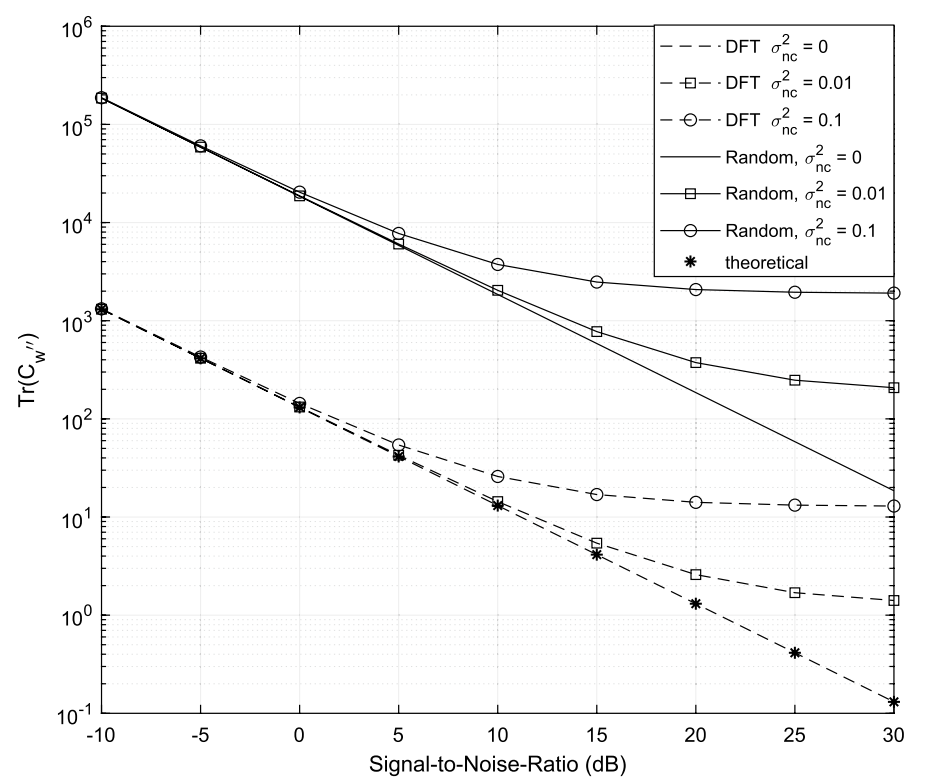

Fig. 4 Total noise power evaluation for a combiner Matrix based on (i) DFT matrix and (ii) random phase shifters for $\sigma_{n c}^{2}=\{0,0.01,0.1\}$

\subsection{Application of full snapshot reconstruction}

In this section, the application of the proposed scheme, under the AoA estimation performance, is evaluated versus the additive noise SNR (see Figs. 5, 6) and the number of required snapshots (see Figs. 7, 8) for the covariance matrix estimation. In the first two experiments, the proposed scheme, called PRE+MUSIC, will be compared with H-MUSIC [23] in the same partially and fully connected hybrid antenna arrays, when the Fourier matrix is utilized, and MUSIC when the conventional antenna array is used. In the last experiment demonstrated in Fig. 9, PRE+MUSIC performance is evaluated for both DFT-based and exponentials with random phases combiner matrix. It is interesting to note that, in the case of the PRE+MUSIC (as well as for the H-MUSIC) a number of $T$ sub-snapshots are needed in order to reconstruct a full conventional snapshot as in (4). Thus, to make a fair comparison with the conventional MUSIC, we have assumed in the experiments that the number $S_{2}$ of snapshots collected by MUSIC is $T$ times the number of snapshots $S_{1}$ used by the proposed scheme and H-MUSIC.

The performance measure that is used, i.e., the RMSE (root-mean-square-error) [45] of the estimated AoAs, is defined as

$$
\operatorname{RMSE}=\sqrt{\frac{\sum_{m_{\mathrm{c}}=1}^{M_{\mathrm{c}}} \sum_{l=1}^{L}\left(\hat{\theta}_{m_{\mathrm{c}}}(l)-\theta(l)\right)^{2}}{L M_{\mathrm{c}}}},
$$

where $\hat{\theta}_{m_{\mathrm{c}}}(l)$ is the estimate of true AoA $\theta(l)$ in the $m_{\mathrm{c}}$ th Monte Carlo trial and $M_{\mathrm{c}}$ is the total number of trials which is set to 500 . Finally, all schemes, in order to estimate the desired AoAs, are employed in the angular range from $-90^{\circ}$ to $90^{\circ}$ which is discretized with a $0.5^{\circ}$ step.

In the context of the first two experiments in Figs. 5, 6, 7 and 8, first, it is assumed that the full antenna array is organized into non-overlapping subarrays of the same 


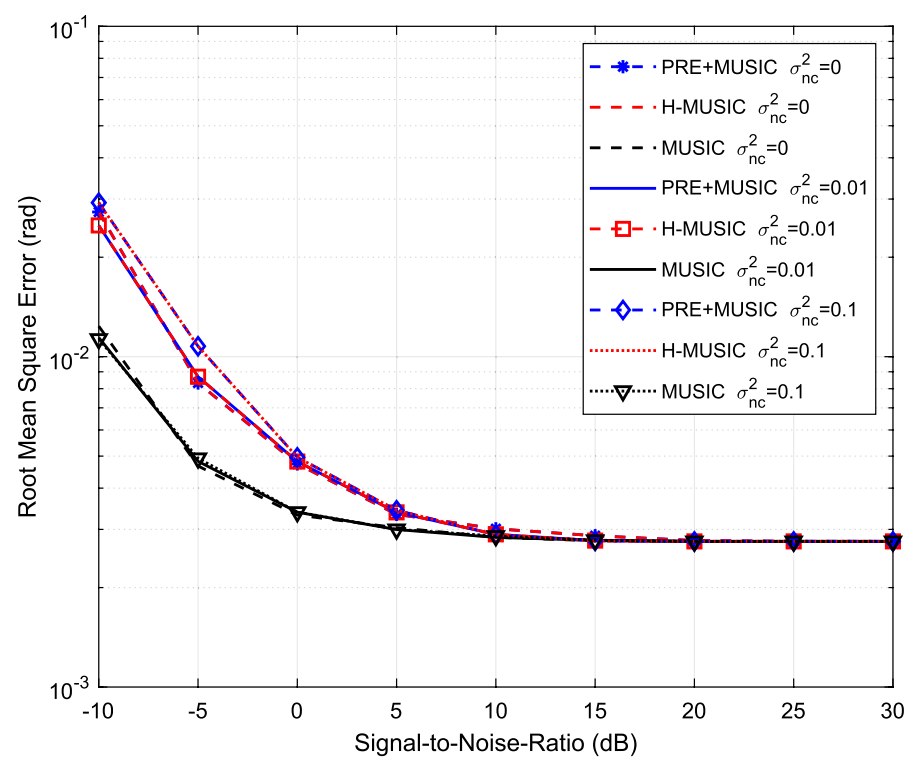

Fig. 5 RMSE of AoA estimation for the partially connected architecture, $M_{C}=500, S_{1}=20, S_{2}=80$, $\lambda=1 \mathrm{~cm}$

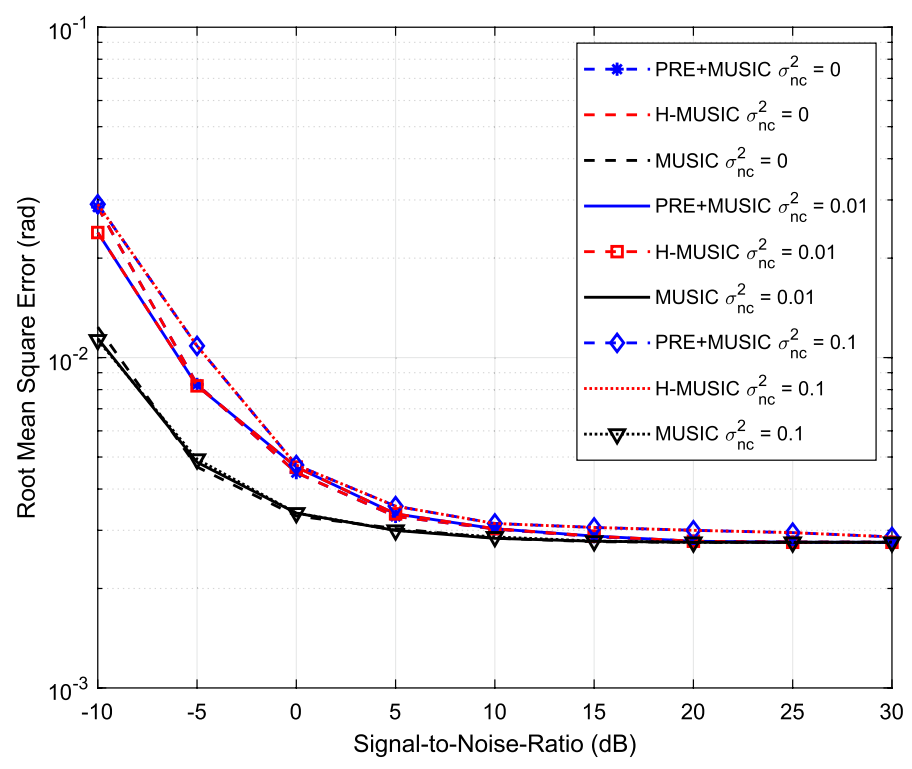

Fig. 6 RMSE of AoA estimation for the fully connected architecture, $M_{c}=500, S_{1}=20, S_{2}=80, \lambda=1 \mathrm{~cm}$

size $M=4$ as in Fig. 2. Specifically, we will demonstrate an experiment assuming that $T=M=4$, i.e., the number of sub-snapshots is equal to the number of antennas in each subarray, and a second experiment considering the fully connected architecture, where all antenna elements contribute to each RF chain, as shown in Fig. 3. In the same experiments of this section, the fully connected architecture, as in Fig. 3, is considered as well, given that $L_{r}=8$ and $T=4$ the desired number of sub-snapshots in order to construct a full snapshot $N=32$. In particular, in Figs. 5 and 6 we demonstrate the 


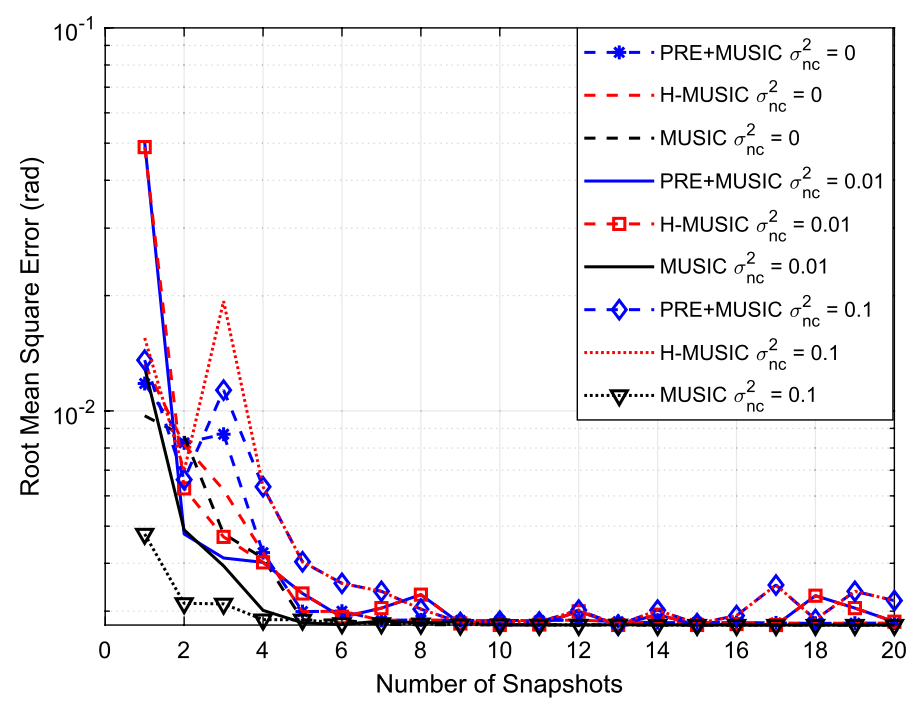

Fig. 7 RMSE of AoA estimation for the partially connected architecture, $M_{c}=500, S N R=20 \mathrm{~dB}, S_{1}=1: 20$, $S_{2}=4: 80$ snapshots $\left(S_{2}=T S_{1}\right.$ where $\left.T=4\right), \lambda=1 \mathrm{~cm}$

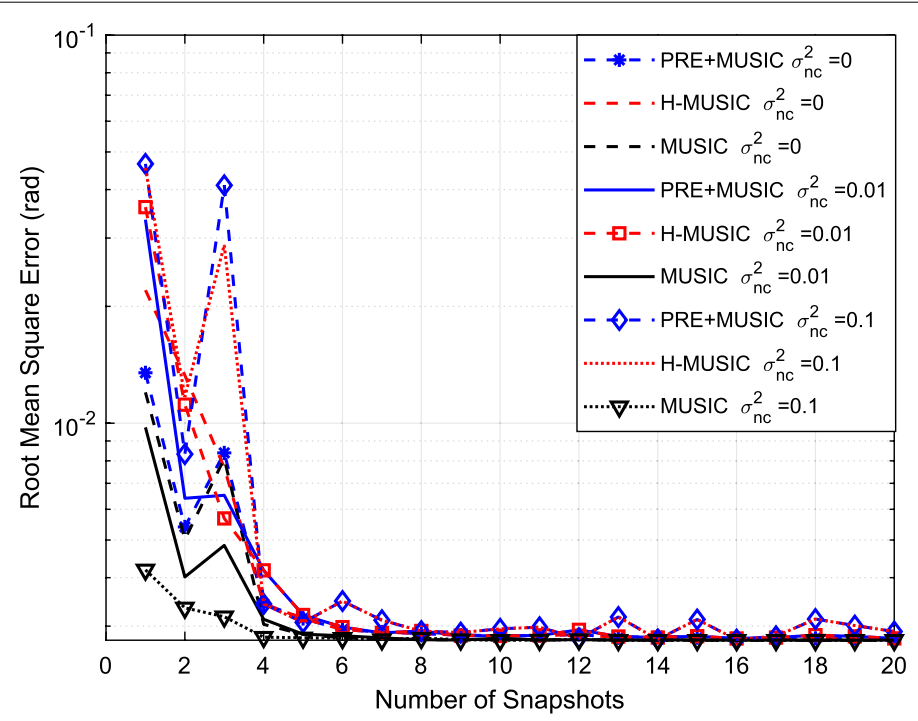

Fig. 8 RMSE of AoA estimation for the fully connected architecture, $M_{C}=500, S N R=20 \mathrm{~dB}, S_{1}=1: 20$, $S_{2}=4: 80$ snapshots $\left(S_{2}=T S_{1}\right.$ where $\left.T=4\right), \lambda=1 \mathrm{~cm}$

RMSE of the three schemes (i.e., PRE+MUSIC, H-MUSIC and MUSIC) versus the SNR for $\sigma_{n c}^{2}=\{0,0.01,0.1\}$ and $S_{1}=20, S_{2}=80$, respectively. It can be observed that both schemes designed for hybrid antennas (i.e., PRE+MUSIC and H-MUSIC) have almost identical performance, which attains that of MUSIC for high SNR values. Also, MUSIC has the best performance at very low SNRs, but it is noted that MUSIC is applicable only to conventional ULAs and, for the same observation time, it utilizes $T$ times more full snapshots, therefore, it is able to have a better estimation of the covariance matrix.

In the context of the second experiment in Figs. 7 and 8, the RMSE performance versus the number of snapshots is also evaluated for $\sigma_{n c}^{2}=\{0,0.01,0.1\}$ and $S N R=20 \mathrm{~dB}$. 


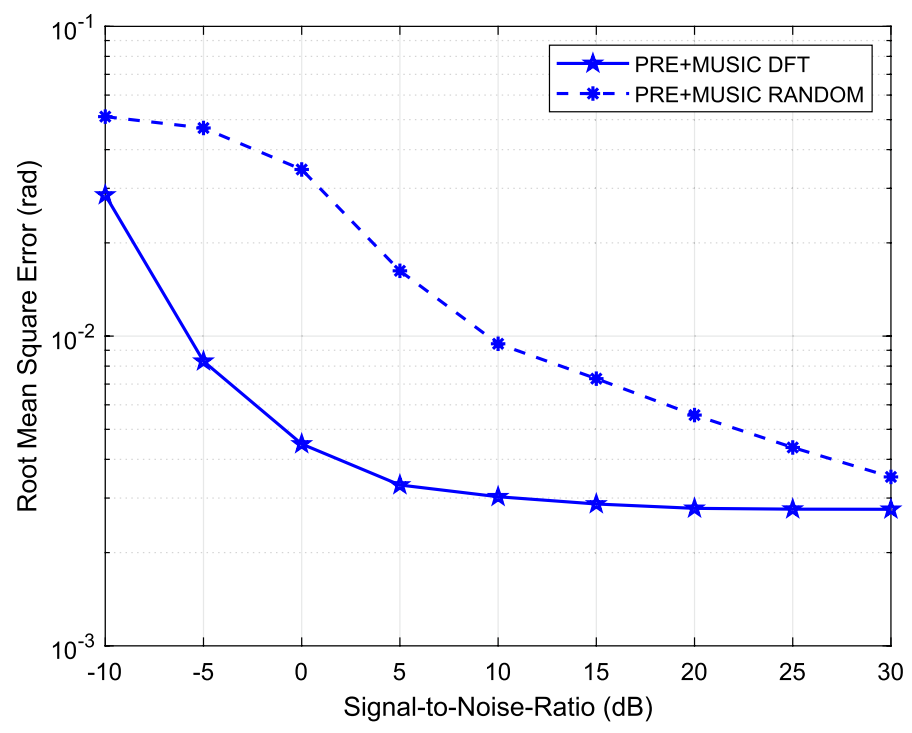

Fig. 9 RMSE of AoA estimation of PREMUSIC for a combiner matrix based on (i) DFT and (ii) random phase shifters, $M_{C}=500, S_{1}=20, S_{2}=80, \lambda=1 \mathrm{~cm}$

Obviously, as illustrated in Figs. 7 and 8, in the case of both architectures and irrespective of the non-constant signals power $\sigma_{n c}^{2}$, the classical MUSIC algorithm can achieve better AoA estimation performance than the rest schemes, as the number of snapshots actually used is larger. Moreover, it is also observed that the RSME performance is similar for all schemes for $S_{1} \geq 8$ or $S_{2} \geq 32$ despite MUSIC captures greater number of snapshots than the hybrid schemes. Also, concerning the source signals time-variations (in both architectures), it is observed that all schemes are not affected in terms of the AoA estimation and their behavior is approximately the same as in the case of constant sources.

Additionally, in the last experiment in Fig. 9 the focus is solely on PRE+MUSIC, at which, it is depicted the impact of a random phase-shift combiner matrix in the AoA estimation. Since, the proposed scheme performance is slightly affected by the possible time variations of the source signals and the hybrid architecture type, this experiment is conducted assuming only the fully connected architecture for constant value sources. As it was expected, the design of the combiner matrix based on DFT optimized the performance of PRE+MUSIC. Indeed, the cost function values and the lower bound impacted by the use of a random phase-shift matrix (as demonstrated in Fig. 4), which impacted its performance under the AoA estimation problem, as well, as shown in Fig. 9. This relates with the fact that, in the reconstructed full snapshot the noise term is not white if the $\mathcal{B}(n)$ is not unitary.

We should refer that the use of orthogonal beamforming matrices, e.g., the use of the discrete Fourier matrix achieves the optimal behavior of the performance metric (RMSE) in terms AoA estimation. Non-orthogonal beamforming matrices, e.g., random beamforming matrices, can be utilized as well. Notwithstanding, the performance in terms of the AoA estimation accuracy may be considerably degraded in comparison with its optimal behavior, as shown in Fig. 9. 
Ultimately, it should be noted that, irrespective of the adopted hybrid architecture and the power of the non-constant signal component, the performance of PRE+MUSIC in terms AoA estimation, is not affected by the floors that appear in the involved cost function in Fig. 4, revealing that the discrete Fourier matrix is an appropriate choice for the design of the RF combiner.

\section{Conclusions}

In summary, we investigated the recoverability of a ULA snapshot applicable to sub- and fully connected hybrid antenna arrays. The recommended scheme is able to recover the full snapshot as if we had a conventional antenna array either for time-varying source signals or constant signals. The phase shifters of the hybrid antenna array were designed with the aim to maximize the STNR of the restored snapshot. Finally, typical simulation results have been presented for the AoA estimation problem, confirming the efficacy of the proposed approach under the optimal solution.

\section{Acknowledgements \\ Not applicable in this section.}

\section{Authors' contributions}

All authors participated in the development of the new methods as well as in the design and execution of the experiments and the interpretation of the obtained results. Also, the authors have jointly contributed to the writing of the manuscript. Finally, all authors read, reviewed and edited the manuscript and have approved the submitted one. All authors read and approved the final manuscript.

\section{Funding}

This research is co-financed by Greece and the European Union (European Social Fund- ESF) through the Operational Programme 'Human Resources Development, Education and Lifelong Learning' in the context of the project 'Strengthening Human Resources Research Potential via Doctorate Research' (MIS-5000432), implemented by the State Scholarships Foundation (IKY). This work is also supported by the European Regional Development Fund and the Republic of Cyprus through the Research and Innovation Foundation under Project INFRASTRUCTURES/1216/0017 IRIDA.

Availability of data and materials

Data sharing not applicable to this article as no datasets were generated or analyzed during the current study.

\section{Competing interests}

The authors declare that they have no competing interests.

Received: 10 June 2020 Accepted: 12 November 2020

Published online: 01 December 2020

\section{References}

1. A. Alkhateeb, J. Mo, N. Gonzalez-Prelcic, R.W. Heath, MIMO precoding and combining solutions for millimeter-wave systems. IEEE Commun. Mag. 52(12), 122-131 (2014)

2. J.A. Zhang, X. Huang, V. Dyadyuk, Y.J. Guo, Massive hybrid antenna array for millimeter-wave cellular communications. IEEE Wirel. Commun. 22(1), 79-87 (2015)

3. R.W. Heath, N. Gonzalez-Prelcic, S. Rangan, W. Roh, A.M. Sayeed, An overview of signal processing techniques for millimeter wave MIMO systems. IEEE J. Sel. Top. Signal Process. 10(3), 436-453 (2016)

4. K. Sakaguchi, T. Haustein, S. Barbarossa, E.C. Strinati, A. Clemente, G. Destino et al., Where, when, and how mm Wave is used in 5 G and beyond. IEICE Trans. Electron. 100(10), 790-808 (2017)

5. S. Ghosh, D. Sen, An inclusive survey on array antenna design for millimeter-wave communications. IEEE Access. 7, 83137-83161 (2019)

6. F. Shu, Y. Qin, T. Liu, L. Gui, Y. Zhang, J. Li et al., Low-complexity and high-resolution DOA estimation for hybrid analog and digital massive MIMO receive array. IEEE Trans. Commun. 66(6), 2487-2501 (2018)

7. M. Majidzadeh, A. Moilanen, N. Tervo, H. Pennanen, A. Tolli, M. Latva-Aho, Partially connected hybrid beamforming for large antenna arrays in multi-user MISO systems, in 28th Annual International Symposium on Personal, Indoor, and Mobile Radio Communications (PIMRC) (2017), pp. 1-6

8. X. Yu, J. Zhang, K.B. Letaief, Partially-connected hybrid precoding in mm-Wave systems with dynamic phase shifter networks. arXiv Preprint (2017). arXiv:170500859

9. R. Méndez-Rial, C. Rusu, N. González-Prelcic, A. Alkhateeb, R.W. Heath, Hybrid MIMO architectures for millimeter wave communications: phase shifters or switches? IEEE Access. 4, 247-267 (2016)

10. M. Xiao, S. Mumtaz, Y. Huang, L. Dai, Y. Li, M. Matthaiou et al., Millimeter wave communications for future mobile networks. IEEE J. Sel. Areas Commun. 35(9), 1909-1935 (2017) 
11. I. Ahmed, H. Khammari, A. Shahid, A. Musa, K.S. Kim, E. De Poorter et al., A survey on hybrid beamforming techniques in 5G: architecture and system model perspectives. IEEE Commun. Surv. Tutor. 20(4), 3060-3097 (2018)

12. L. Li, F. Chen, J. Dai, Separate DOD and DOA estimation for bistatic MIMO radar. Int. J. Antennas Propagat. 2016, 1 (2016)

13. S. Ejaz, M.A. Shafiq, Comparison of spectral and subspace algorithms for FM source estimation. Prog. Electromagn. Res. 14, 11-21 (2010)

14. I. El Ouargui, S. Safi, M. Frikel, Minimum array elements for resolution of several direction of arrival estimation methods in various noise-level environments. J. Telecommun. Inf. Technol. 2, 87-94 (2018)

15. R. Cao, B. Liu, F. Gao, X. Zhang, A low-complex one-snapshot DOA estimation algorithm with massive ULA. IEEE Commun. Lett. 21(5), 1071-1074 (2017)

16. Y. Han, B. Cao, W. Dong, Q. Fang, W. Zhang, An improved mode-music algorithm for DOA estimation of coherent sources based on hybrid array, in 12th International Conference on Signal Processing (ICSP) (2014), pp. 358-362

17. Y. Khmou, S. Safi, M. Frikel, Comparative study between several direction of arrival estimation methods. J. Telecommun. Inf. Technol. 1, 41-48 (2014)

18. T.B. Lavate, V. Kokate, A. Sapkal, Performance analysis of MUSIC and ESPRIT DOA estimation algorithms for adaptive array smart antenna in mobile communication, in 2nd International Conference on Computer and Network Technology (ICCNT) (2010), pp. 308-311

19. F.M. Han, X.D. Zhang, An ESPRIT-like algorithm for coherent DOA estimation. IEEE Antennas Wirel. Propag. Lett. 4(1), 443-446 (2005)

20. R. Roy, T. Kailath, ESPRIT-estimation of signal parameters via rotational invariance techniques. IEEE Trans. Acoust. Speech Signal Process. 37(7), 984-995 (1989)

21. T.J. Shan, M. Wax, T. Kailath, On spatial smoothing for direction-of-arrival estimation of coherent signals. IEEE Trans. Acoust. Speech Signal Process. 33(4), 806-811 (1985)

22. S.U. Pillai, B.H. Kwon, Forward/backward spatial smoothing techniques for coherent signal identification. IEEE Trans. Acoust. Speech Signal Process. 37(1), 8-15 (1989)

23. S.F. Chuang, W.R. Wu, Y.T. Liu, High-resolution AoA estimation for hybrid antenna arrays. IEEE Trans. Antennas Propag. 63(7), 2955-2968 (2015)

24. M. Trigka, C. Mavrokefalidis, K. Berberidis, An effective preprocessing scheme for DoA estimation in hybrid antenna arrays, in 25th International Conference on Telecommunications (ICT) (2018), pp. 127-131

25. M. Trigka, C. Mavrokefalidis, K. Berberidis, AoA estimation scheme for fully-connected hybrid architecture antenna arrays, in 13th European Conference on Antennas and Propagation (EuCAP) (2019), pp. 1-5

26. Z. Ni, J.A. Zhang, K. Yang, F. Gao, J. An, Estimation of multiple angle-of-arrivals with localized hybrid subarrays for millimeter wave systems. IEEE Trans. Commun. 68, 3 (2019)

27. R. Zhang, J. Zhou, J. Lan, B. Yang, Z. Yu, A high-precision hybrid analog and digital beamforming transceiver system for 5 G millimeter-wave communication. IEEE Access. 7, 83012-83023 (2019)

28. C. Qin, J.A. Zhang, X. Huang, K. Wu, Y.J. Guo, Fast angle-of-arrival estimation via virtual subarrays in analog antenna array. IEEE Trans. Wirel. Commun. 19, 6425-6439 (2020)

29. K. Wu, W. Ni, T. Su, R.P. Liu, Y.J. Guo, Recent breakthroughs on angle-of-arrival estimation for millimeter-wave highspeed railway communication. IEEE Commun. Mag. 57(9), 57-63 (2019)

30. K.C. Hwang, A modified Sierpinski fractal antenna for multiband application. IEEE Antennas Wirel. Propag. Lett. 6 $357-360(2007)$

31. E. Guariglia, Entropy and fractal antennas. Entropy 18(3), 84 (2016)

32. E. Guariglia, Spectral analysis of the weierstrass-mandelbrot function, in 2017 2nd International Multidisciplinary Conference on Computer and Energy Science (SpliTech) (IEEE, New York, 2017), pp. 1-6

33. X. Zheng, Y.Y. Tang, J. Zhou, A framework of adaptive multiscale wavelet decomposition for signals on undirected graphs. IEEE Trans. Signal Process. 67(7), 1696-1711 (2019)

34. C. Puente-Baliarda, J. Romeu, R. Pous, A. Cardama, On the behavior of the Sierpinski multiband fractal antenna. IEEE Trans. Antennas Propag. 46(4), 517-524 (1998)

35. E. Guariglia, Primality, fractality, and image analysis. Entropy 21(3), 304 (2019)

36. J. Anguera, C. Borja, C. Puente, Microstrip fractal-shaped antennas: a review, in The 2nd European Conference on Antennas and Propagation, EUCAP 2007. IET (2007), pp. 1-7

37. E. Guariglia, Harmonic Sierpinski gasket and applications. Entropy 20(9), 714 (2018)

38. S.R. Best, A discussion on the significance of geometry in determining the resonant behavior of fractal and other non-Euclidean wire antennas. IEEE Antennas Propag. Mag. 45(3), 9-28 (2003)

39. E. Guariglia, S. Silvestrov, Fractional-wavelet analysis of positive definite distributions and wavelets on $\mathscr{D}{ }^{\prime}(\mathbb{C})$, in Engineering Mathematics II (Springer, Berlin, 2016), pp. 337-353

40. C. Zhou, Y. Gu, S. He, Z. Shi, A robust and efficient algorithm for coprime array adaptive beamforming. IEEE Trans. Veh. Technol. 67(2), 1099-1112 (2017)

41. E. Björnson, L. Sanguinetti, H. Wymeersch, J. Hoydis, T.L. Marzetta, Massive MIMO is a reality-What is next? Five promising research directions for antenna arrays. Digit. Signal Proc. 94, 3-20 (2019)

42. O. El Ayach, S. Rajagopal, S. Abu-Surra, Z. Pi, R.W. Heath, Spatially sparse precoding in millimeter wave MIMO systems. IEEE Trans. Wirel. Commun. 13(3), 1499-1513 (2014)

43. R. Dinis, A. Palhau, A class of signal-processing schemes for reducing the envelope fluctuations of CDMA signals. IEEE Trans. Commun. 53(5), 882-889 (2005)

44. A.W. Marshall, Inequalities: Theory of Majorization and Its Applications (Academic Press, London, 1979)

45. S. Ren, X. Ma, S. Yan, C. Hao, 2-D unitary ESPRIT-like direction-of-arrival (DoA) estimation for coherent signals with a uniform rectangular array. Sensors 13(4), 4272-4288 (2013)

\section{Publisher's Note}

Springer Nature remains neutral with regard to jurisdictional claims in published maps and institutional affiliations. 